\title{
Theoretical Study on the Mechanism of
} Aggregation Caused Quenching in Near-Infrared Thermally Activated Delayed Fluorescence Molecules: Hydrogen-Bond Effect

Kai Zhang, Jie Liu, Yuchen Zhang, Jianzhong Fan, Chuan-Kui Wang*, Lili Lin* Shandong Province Key Laboratory of Medical Physics and Image Processing Technology, School of Physics and Electronics, Shandong Normal University, 250014 Jinan, China.

\section{Corresponding Author}

* Author to whom correspondence should be addressed. E-mail:

ckwang@sdnu.edu.cn and linll@sdnu.edu.cn . 


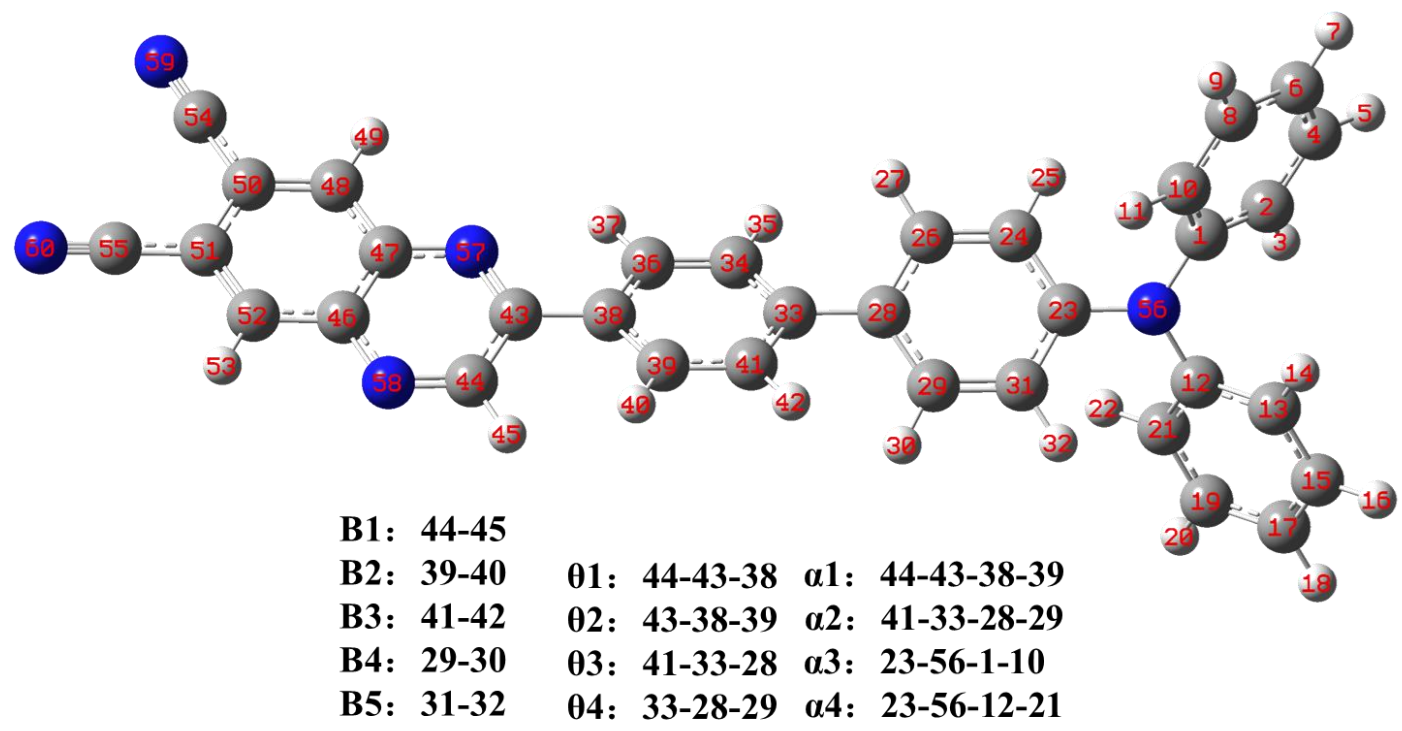

Figure S1. The atomic labels and the interesting bond lengths (B1, B2, B3, B4 and B5), bond angles ( $(\theta 1, \theta 2, \theta 3$ and $\theta 4)$ and dihedral angles $(\alpha 1, \alpha 2, \alpha 3$ and $\alpha 4)$.
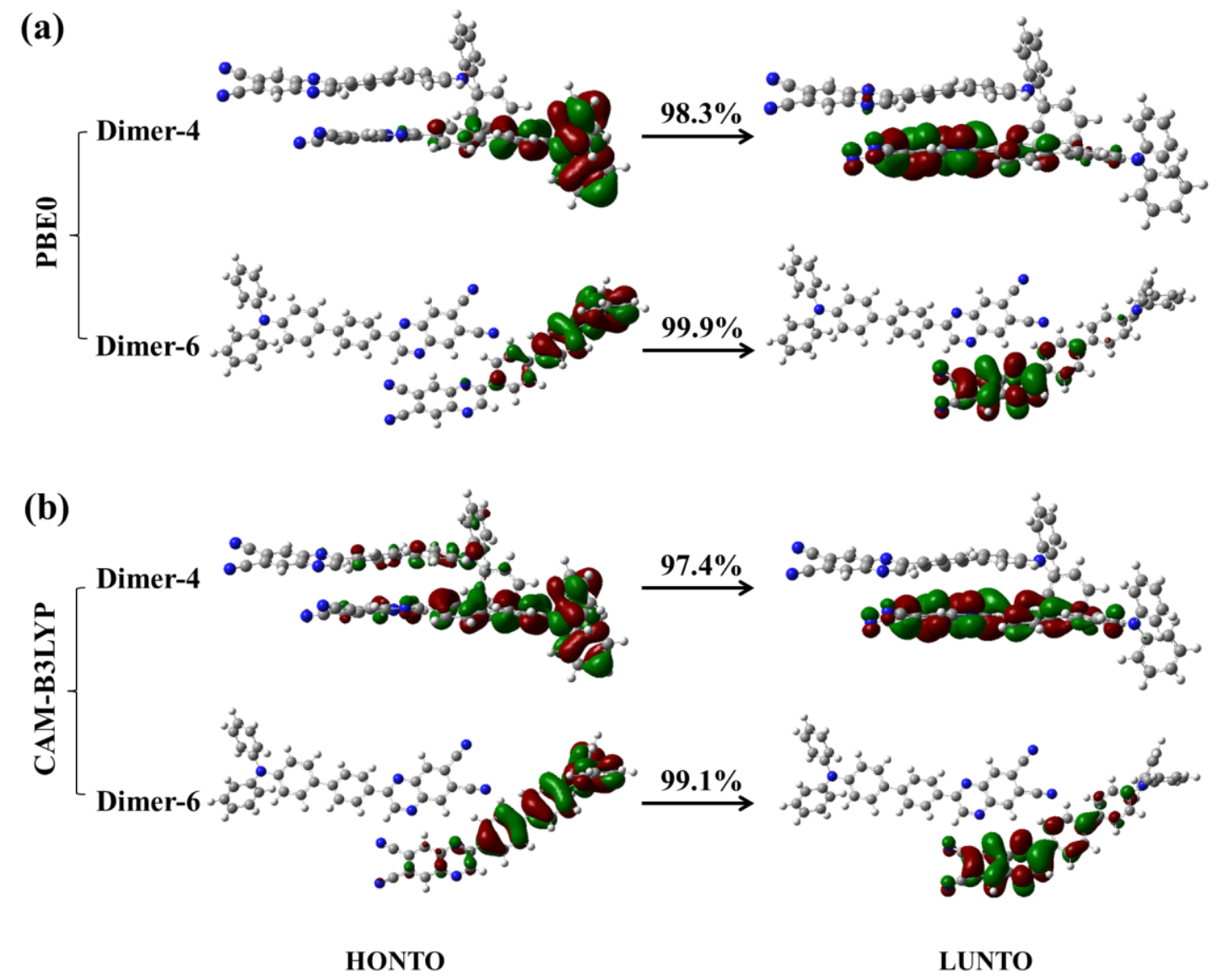

Figure S2. Electron distribution in transition orbitals of Dimer-6 and Dimer-4 calculated with QM/MM calculation where the PBE0 functional and the Cam-B3LYP functional is adopted for the QM calculation respectively. (isovalue=0.02). 


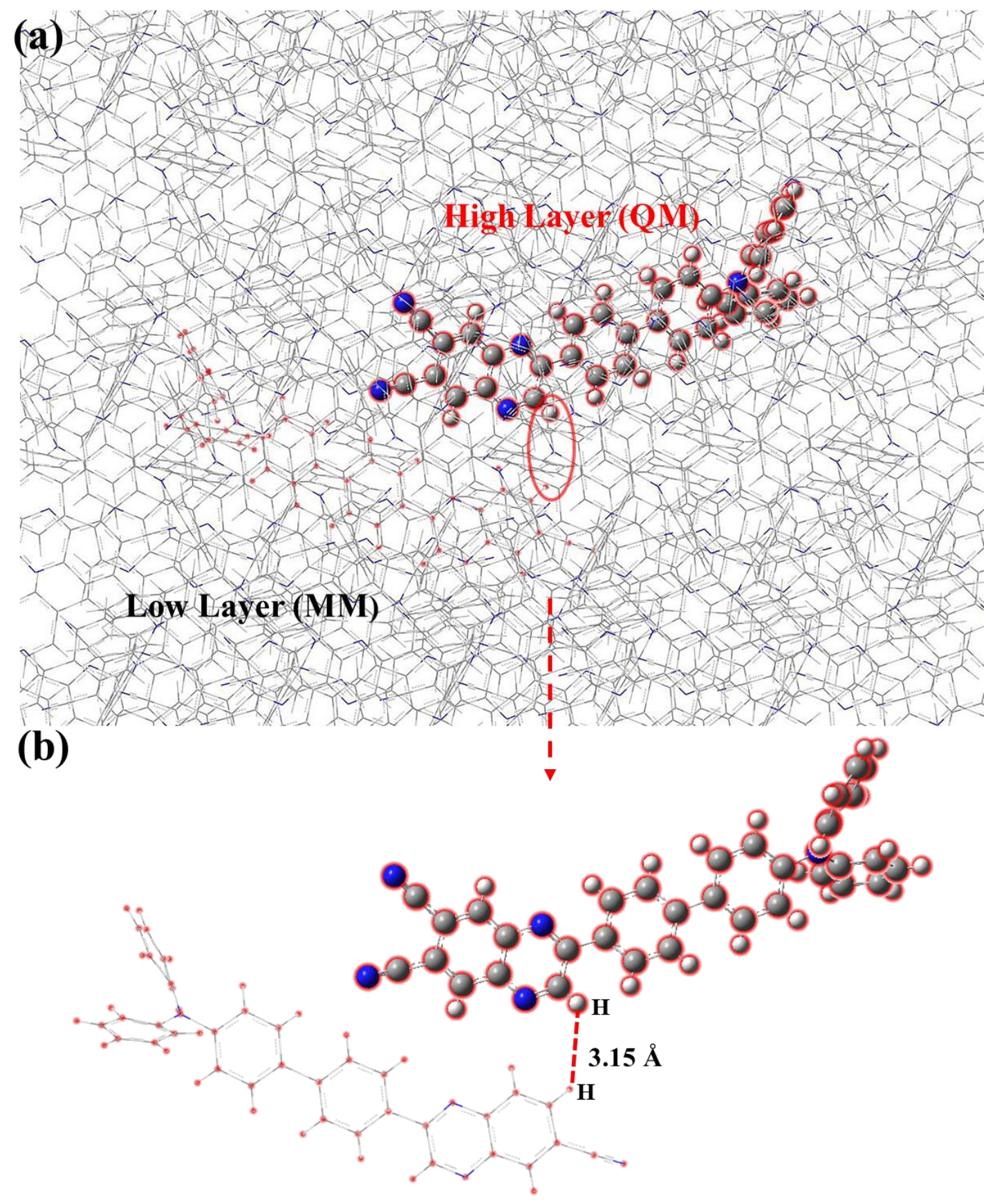

Figure S3. (a) ONIOM model: the centered molecule is treated as a high layer and the surrounding molecules are fixed as a low layer. (b) Dimer-6 without hydrogen bond. 

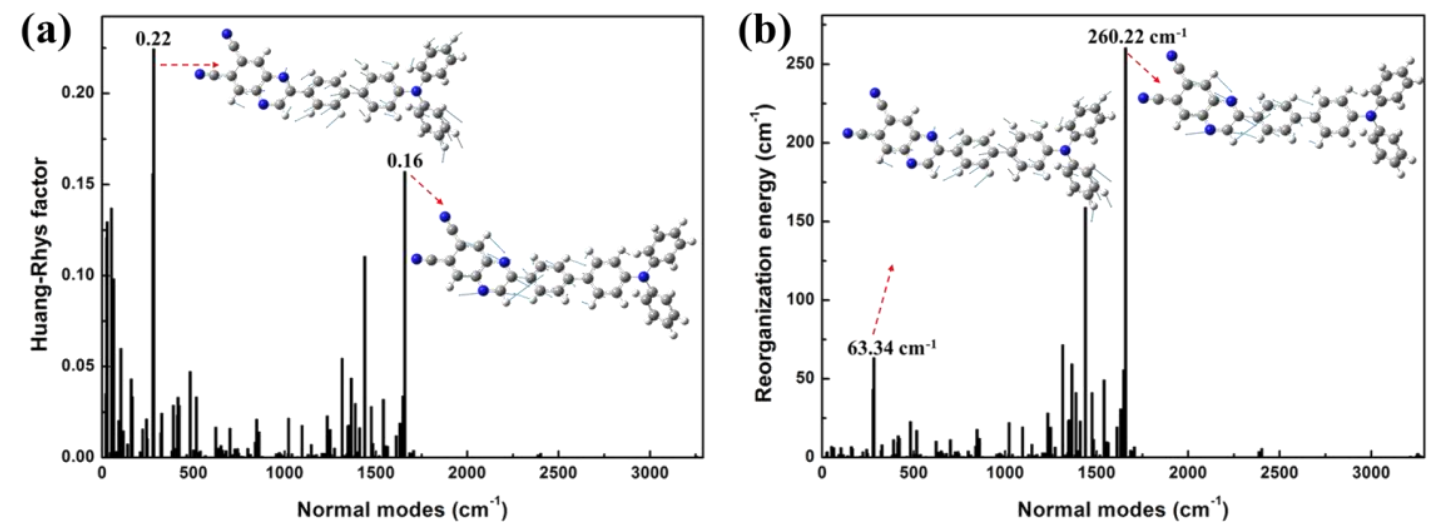

Figure S4. Calculated HR factors (a) and the reorganization energies (b) versus the normal mode frequencies for the model without hydrogen bond in aggregation state. Representative vibration modes are shown as insets.

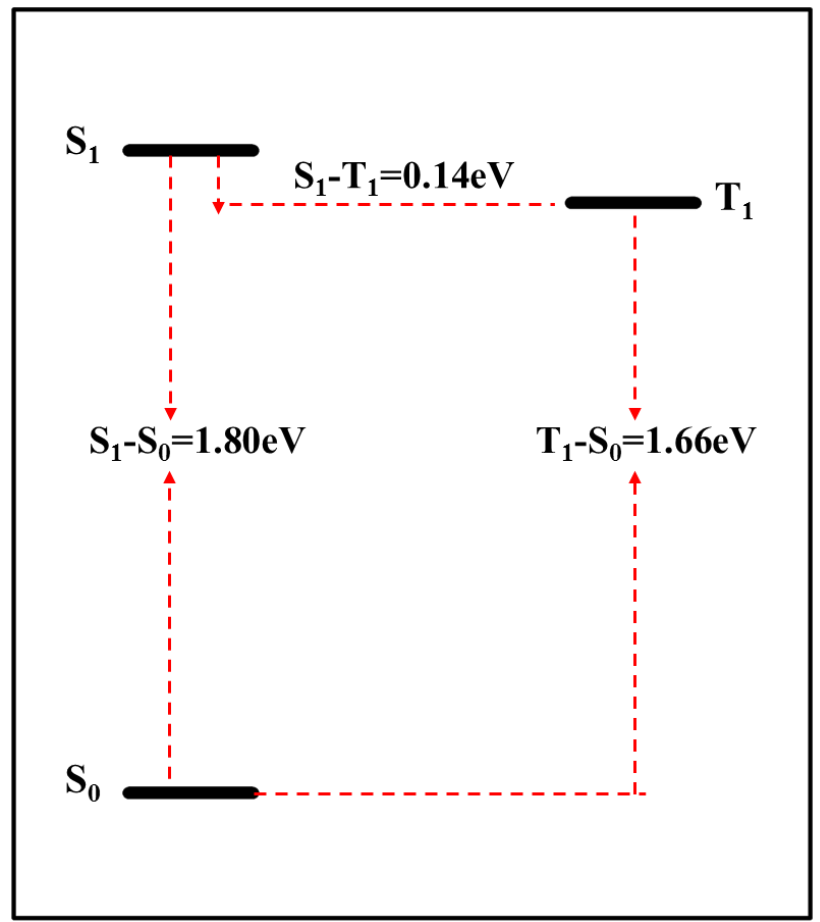

Figure S5. Energy levels of low-lying excited states of the TPA-QCN molecule in aggregation state without hydrogen bond. 

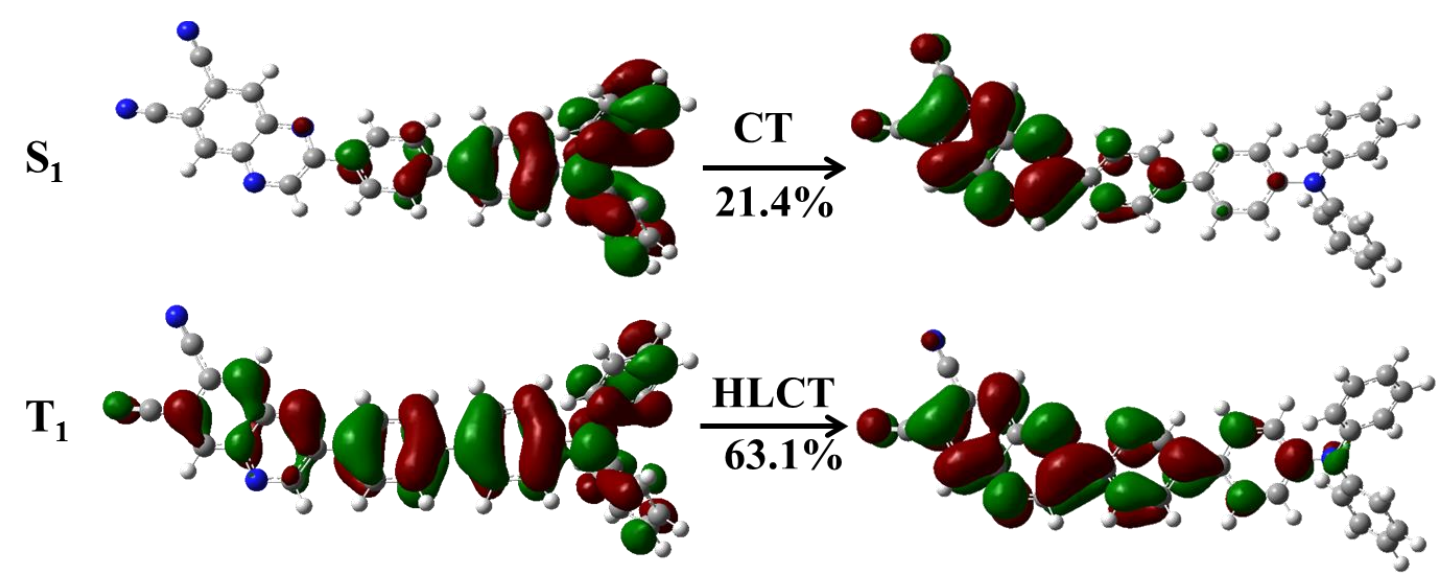

HONTO

LUNTO

Figure S6. Electron distribution of HONTO and LUNOT for $S_{1}$ and $T_{1}$ of the TPA-QCN molecule in aggregation without hydrogen bond.

Table S1. Selected Bond Lengths (in $\AA$ ), Angles, and Torsion Angles (in degree) of the $\mathrm{S}_{0}$ and $\mathrm{S}_{1}$ for TPA-QCN in toluene and in aggregation state.

\begin{tabular}{cccc|ccc}
\hline & \multicolumn{3}{|c|}{ Toluene } & \multicolumn{3}{|c}{ Aggregation } \\
& $\mathrm{S}_{0}$ & $\mathrm{~S}_{1}$ & $\Delta\left(\mathrm{S}_{1}-\mathrm{S}_{0}\right)$ & $\mathrm{S}_{0}$ & $\mathrm{~S}_{1}$ & $\Delta\left(\mathrm{S}_{1}-\mathrm{S}_{0}\right)$ \\
\hline B1 & 1.087 & 1.088 & 0.001 & 1.073 & 1.074 & 0.001 \\
$\mathbf{B 2}$ & 1.086 & 1.086 & 0 & 1.085 & 1.084 & -0.001 \\
$\mathbf{B 3}$ & 1.086 & 1.087 & 0.001 & 1.086 & 1.086 & 0 \\
$\mathbf{B 4}$ & 1.087 & 1.085 & -0.002 & 1.087 & 1.086 & -0.001 \\
$\mathbf{B 5}$ & 1.085 & 1.085 & 0 & 1.086 & 1.086 & 0 \\
$\boldsymbol{\theta 1}$ & 121.562 & 122.339 & 0.777 & 120.255 & 119.683 & -0.572 \\
$\boldsymbol{\theta 2}$ & 122.339 & 122.876 & 0.537 & 122.951 & 122.317 & -0.634 \\
$\boldsymbol{\theta 3}$ & 121.157 & 120.935 & -0.222 & 123.579 & 122.988 & -0.591 \\
$\boldsymbol{\theta 4}$ & 121.335 & 121.038 & -0.297 & 122.910 & 122.580 & -0.33 \\
$\boldsymbol{\alpha 1}$ & -16.913 & -18.104 & -1.191 & -12.420 & -25.013 & -12.593 \\
$\boldsymbol{\alpha 2}$ & 33.729 & 36.630 & 2.901 & 26.703 & 27.956 & 1.253 \\
$\boldsymbol{\alpha 3}$ & 43.127 & 36.918 & -6.209 & 33.224 & 28.304 & -4.92 \\
$\boldsymbol{\alpha 4}$ & 43.348 & 36.963 & -6.385 & 50.405 & 44.264 & -6.141 \\
\hline
\end{tabular}


Table S2. Spin-orbit coupling (SOC) constants between $S_{1}$ and $T_{1}$ of the model without hydrogen bond, the intersystem crossing rate and reverse intersystem crossing rate. The radiative rate, the internal conversion rate and the fluorescence rate are also listed.

\begin{tabular}{lccccccc}
\hline & $\begin{array}{c}\mathrm{SOC}^{\mathrm{a}} \\
\left(\mathrm{cm}^{-1}\right)\end{array}$ & $\begin{array}{c}k_{I S C} \\
\left(\mathrm{~s}^{-1}\right)\end{array}$ & $\begin{array}{c}\mathrm{SOC}^{\mathrm{b}} \\
\left(\mathrm{cm}^{-1}\right)\end{array}$ & $\begin{array}{c}k_{R I S C} \\
\left(\mathrm{~s}^{-1}\right)\end{array}$ & $\begin{array}{c}k_{r} \\
\left(\mathrm{~s}^{-1}\right)\end{array}$ & $\begin{array}{c}k_{I C} \\
\left(\mathrm{~s}^{-1}\right)\end{array}$ & $\Phi_{\mathrm{PF}}$ \\
\hline $\mathrm{S}_{1}-\mathrm{S}_{0}$ & ---- & ---- & --- & --- & $2.00 \times 10^{7}$ & $1.72 \times 10^{8}$ & $9.36 \%$ \\
$\mathrm{~S}_{1}-\mathrm{T}_{1}$ & 0.202 & $2.17 \times 10^{7}$ & 0.226 & $9.25 \times 10^{4}$ & ---- & --- & --- \\
\hline
\end{tabular}

Notes: a. calculated based on the optimized geometry of $S_{1}$.

b. calculated based on the optimized geometry of $\mathrm{T}_{1}$. 